\title{
EVALUACIÓN DE LOS HERBICIDAS CROSSER Y VAQUERO EN EL CONTROL DE MALEZAS HERBÁCEAS EN PASTIZALES TROPICALES1
}

\author{
Valentín Esqueda ${ }^{2}$
}

\begin{abstract}
RESUMEN
Evaluación de los herbicidas Crosser y Vaquero en el control de malezas herbáceas en pastizales tropicales. Durante la estación lluviosa de 2001, se establecieron dos experimentos en La Mancha, mpio. de Actopan, Ver., México, para evaluar el efecto de los herbicidas Crosser (15 g de picloram + $150 \mathrm{~g}$ de 2,4-D/L) y Vaquero (30 g de triclopir + $120 \mathrm{~g}$ de 2,4-D/L) en el control de la hierba ceniza (Lagascea mollis Cav.) y la flor amarilla (Baltimora recta L.) en un pastizal de zacate Estrella de Africa (Cynodon plectostachyus Vanderyst). Los experimentos se repitieron en 2002, en La Colonia Independencia, mpio. de Tres Valles, Ver., en un pastizal de zacate Pangola (Digitaria decumbens Stent.), infestado con frijolillo [Senna obtusifolia (L.) Irwin \& Barneby]. Tanto el Crosser como el Vaquero se aplicaron a las dosis de 0,$75 ; 1 ; 1,25 ; 1,5$ y $21 / 1001$ de agua y se utilizaron como testigos a Full-Mina 4 (480 g de 2,4-D/L) a 1 y 2 1/100 1 de agua y Esterón 47-M (400 g de 2,4-D/L) a 2 1/100 1 de agua; un testigo sin aplicar también fue incluido. En todos los experimentos se utilizó un diseño experimental de bloques al azar con cuatro repeticiones. El control de las malezas y la toxicidad a los pastos se evaluó a los 15, 30 y 60 días después de la aplicación de los tratamientos. El Crosser controló a la flor amarilla y al frijolillo desde 0,75 1/100 l de agua, mientras que para controlar eficientemente a la hierba ceniza se necesitó aplicarlo a 1,5 1/100 l de agua. El Vaquero también controló a la flor amarilla y al frijolillo a partir de 0,75 1/100 l de agua, pero no tuvo un buen control de la hierba ceniza. Ninguno de los tratamientos ocasionó toxicidad a los pastos.
\end{abstract}

\begin{abstract}
Evaluation of the herbicides Crosser and Vaquero for weed control in tropical grasslands. During the 2001 rainy season, two experiments were conducted in La Mancha, Veracruz, Mexico, in order to evaluate the effect of the herbicides Crosser ( $15 \mathrm{~g}$ of picloram $+150 \mathrm{~g}$ of 2,4-D/L) and Vaquero (30 $\mathrm{g}$ of triclopyr $+120 \mathrm{~g}$ of 2,4-D/L) for controlling Lagascea mollis Cav. and Baltimora recta $\mathrm{L}$. in a grassland of Cynodon plectostachyus Vanderyst. In 2002, the experiments were repeated in La Colonia Independencia, Ver., in a grassland of Digitaria decumbens Stent., infested with Senna obtusifolia (L.) Irwin \& Barneby. Both herbicides, Crosser and Vaquero were applied at $0.75,1,1.25,1.5$ and $21 / 1001$ of water; Full-Mina 4 (480 $\mathrm{g}$ of 2,4-D/L) at 1 and 2 1/100 1 of water, and Esteron $47-\mathrm{M}(400 \mathrm{~g}$ of 2,4-D/L) at $21 / 1001$ of water, were also evaluated and a weeded control was included. A Completely Randomized Block Design with four replications was used for all the experiments. Weed control and grass toxicity were evaluated at 15, 30 and 60 days after treatment application. Crosser controlled $B$. recta and $S$. obtusifolia at $0.751 / 1001$ of water, whereas to control $L$. mollis, it was necessary to apply it at 1.5 1/100 1 of water. Vaquero also controlled $B$. recta and $S$. obtusifolia at 0.75 1/100 1 of water, but it had no good control of L. mollis. None of the herbicide treatments caused any toxicity to the grasses.
\end{abstract}

\section{INTRODUCCIÓN}

En el estado de Veracruz, México, se tienen alrededor de 3,6 millones de hectáreas de pastizales que se dedican a la explotación extensiva del ganado bovino; a nivel nacional, la entidad veracruzana, ocupa el primer lugar en la producción de carne de bovinos y el quinto en producción de leche (Juárez et al. 2000).

\footnotetext{
1 Recibido para publicación en abril del 2003. Presentado en la XLIX Reunión Anual del PCCMCA, La Ceiba, Honduras 2003.

2 Campo Experimental Cotaxtla. CIRGOC. INIFAP. SAGARPA.
} 
Un manejo adecuado de los pastizales es esencial para obtener mayor cantidad y mejor calidad de los pastos, lo cual se refleja con el tiempo de engorda del ganado y en la economía del productor. Uno de los principales factores a considerar en los pastizales, es la eliminación de las malezas, ya que si éstas no son controladas con oportunidad y eficiencia, aprovechan el agua, los nutrimentos y la luz, disminuyendo la disponibilidad a los pastos (Koppel et al. 1999), asimismo, reducen el área de pastoreo aprovechable, pueden causar heridas o toxicidad al ganado y son refugio de fauna indeseable (Avila 1988, Silva et al. 1990).

El estado de Veracruz se caracteriza por tener diversas condiciones agroecológicas, por lo que el espectro de malezas que se presentan en los pastizales es muy amplio. Por lo general, las principales especies, son plantas dicotiledóneas, también conocidas como malezas de hoja ancha, de las cuales pueden coexistir especies leñosas, semileñosas y herbáceas (Enríquez et al. 1999).

La quema, los chapeos manuales o mecánicos y la aplicación de herbicidas selectivos son los métodos más utilizados para el control de las malezas en los pastizales (Hernández y Reichert 1987). Los chapeos solamente proporcionan un control temporal y deben repetirse con cierta periodicidad durante la temporada lluviosa (Radillo y Nava 2001, Reichert 1986). En la mayoría de los casos, el control químico es más eficiente que la quema o los chapeos, ya que paulatinamente se logra la eliminación de las malezas o una disminución significativa de sus poblaciones (Motooka 1986, Esqueda 2000). En la actualidad, los herbicidas Tordón 101 (64 g de picloram + $240 \mathrm{~g}$ de 2,4-D/L) y Arbuskip (80 g de picloram $+80 \mathrm{~g}$ de fluroxipir/L) son ampliamente utilizados para el control de las malezas sobre todo las de tipo arbustivo.

Cuando en el pastizal solamente se presentan malezas herbáceas, la aplicación de los herbicidas antes mencionados, puede tener un costo elevado. En la actualidad se están desarrollando nuevos herbicidas como el Crosser y el Vaquero con los cuales el control de las malezas herbáceas puede resultar más económico, por lo que se evaluó su efecto, sobre tres de las principales especies de malezas herbáceas de los pastizales del estado de Veracruz.

\section{Objetivos}

Evaluar la efectividad de los herbicidas Crosser y Vaquero en el control de malezas herbáceas en potreros y determinar si estos herbicidas ocasionan toxicidad a los pastos Estrella de Africa y Pargola.

\section{MATERIALES Y MÉTODOS}

En la estación lluviosa (temporal) de 2001 se establecieron dos experimentos en una pradera de zacate Estrella de Africa (Cynodon plectostachyus Vanderyst), en La Mancha, Mpio. de Actopan, Ver., en un experimento se evaluó el herbicida Crosser y en otro, el herbicida Vaquero. A su vez, en el ciclo de temporal de 2002, ambos experimentos se establecieron en un potrero de zacate Pangola (Digitaria decumbens Stent.), en La Colonia Independencia, Mpio. de Tres Valles, Ver.

Los herbicidas evaluados fueron: Crosser (concentrado soluble de $15 \mathrm{~g}$ de picloram $+150 \mathrm{~g}$ de 2,4-D/L) y Vaquero, (concentrado emulsionable de $30 \mathrm{~g}$ de triclopir + $120 \mathrm{~g}$ de 2,4-D/L). Se incluyeron Full-Mina 4 (solución concentrada acuosa de $480 \mathrm{~g}$ de 2,4-D/L y Esterón 47-M (concentrado emulsionable de $400 \mathrm{~g}$ de 2,4$\mathrm{D} / \mathrm{L})$ como testigos regionales.

Tanto en 2001, como en 2002, en cada experimento se evaluaron nueve tratamientos, los cuales se indican en los Cuadros 1 y 2.

Cuadro 1. Descripción de los tratamientos del experimento para evaluar el herbicida Crosser. Veracruz, México. 2001.

\begin{tabular}{|c|c|c|c|}
\hline Tratamientos & $\begin{array}{c}\text { Dosis } \\
\text { (1/100 l } \\
\text { agua) }\end{array}$ & $\begin{array}{c}\text { Dosis } \\
\text { (g i.a.*/100 l } \\
\text { agua) }\end{array}$ & $\begin{array}{c}\text { Época } \\
\text { de aplicación }\end{array}$ \\
\hline 1. Crosser & 0,75 & $11,25+112,5$ & Postemergencia \\
\hline 2. Crosser & 1 & $15+150$ & Postemergencia \\
\hline 3. Crosser & 1,25 & $18,75+187,5$ & Postemergencia \\
\hline 4. Crosser & 1,5 & $22,5+225$ & Postemergencia \\
\hline 5. Crosser & 2 & $30+300$ & Postemergencia \\
\hline 6. Full-Mina 4 & 1 & 480 & Postemergencia \\
\hline 7. Full-Mina 4 & 2 & 960 & Postemergencia \\
\hline 8. Esterón 47-M & 1 & 400 & Postemergencia \\
\hline $\begin{array}{l}\text { 9. Testigo sin } \\
\text { aplicar }\end{array}$ & - & - & - \\
\hline
\end{tabular}

Los tratamientos fueron distribuídos en el terreno de acuerdo a un diseño experimental de bloques completos al azar con cuatro repeticiones. Las parcelas experimentales tuvieron un tamaño de cinco $\mathrm{m}$ de largo $\mathrm{x}$ cuatro $\mathrm{m}$ de ancho, equivalente a una superficie de $20 \mathrm{~m}^{2}$.

En el temporal de 2001, los herbicidas se aplicaron el 7 de agosto; al momento de la aplicación, las malezas dominantes tenían una altura de entre 20 y $45 \mathrm{~cm}$, con un promedio de $23,5 \mathrm{~cm}$. A su vez, en el temporal 
Cuadro 2. Descripción de los tratamientos del experimento para evaluar el herbicida Vaquero. Veracruz, México. 2001.

\begin{tabular}{llcl}
\hline Tratamientos & $\begin{array}{c}\text { Dosis } \\
\text { (1/100 I } \\
\text { agua) }\end{array}$ & $\begin{array}{c}\text { Dosis } \\
\text { (g i.a.*/100 I } \\
\text { agua) }\end{array}$ & $\begin{array}{c}\text { Época } \\
\text { de aplicación }\end{array}$ \\
\hline 1. Vaquero & 0,75 & $22,5+90$ & Postemergencia \\
2. Vaquero & 1 & $30+120$ & Postemergencia \\
3. Vaquero & 1,25 & $37,5+150$ & Postemergencia \\
4. Vaquero & 1,5 & $45+180$ & Postemergencia \\
5. Vaquero & 2 & $60+240$ & Postemergencia \\
6. Full-Mina 4 & 1 & 480 & Postemergencia \\
7. Full-Mina 4 & 2 & 960 & Postemergencia \\
8. Esterón 47-M & 1 & 400 & Postemergencia \\
9. Testigo sin & & & \\
\multicolumn{1}{c}{ aplicar } & - & - & - \\
\hline
\end{tabular}

*g.i.a.= gramos ingrediente activo.

de 2002, la aplicación se realizó el 1 de julio, cuando la especie dominante de maleza tenía una altura de entre 40 y $70 \mathrm{~cm}$, con un promedio de $58 \mathrm{~cm}$. En los cuatro experimentos, se utilizó una aspersora manual de mochila con capacidad de 121 , equipada con una boquilla Lurmark 04 F110. La solución herbicida se asperjó de manera de cubrir completamente las malezas, pero sin llegar al escurrimiento. En las parcelas correspondientes al testigo sin aplicar, se permitió a la maleza desarrollarse libremente durante el período de evaluación del experimento.

La densidad de población de malezas se determinó al momento de la aplicación de los tratamientos de cada experimento. Para esto, se utilizó un cuadro de 1 x 1 $\mathrm{m}$, el cual fue lanzado al azar en las parcelas correspondientes a los testigos sin aplicar. Las malezas fueron identificadas y cuantificadas, y se hicieron las transformaciones necesarias para reportar su densidad en número de individuos por hectárea.
Las evaluaciones de control de malezas se llevaron a cabo a los 15, 30 y 60 días después de la aplicación de los tratamientos (DDA), Se evaluó visualmente el efecto de los herbicidas sobre las especies de malezas dominantes, en la totalidad de cada parcela experimental. Para evaluar se utilizó la escala porcentual (0 - 100\%), en donde 0 significó que la maleza no fue afectada y $100 \%$ que fue completamente eliminada.

En las mismas fechas de evaluación del control de malezas, se evaluó visualmente la toxicidad a los pastos y se asignaron valores en la escala de 0 a $100 \%$, en donde 0 , significó que el pasto no fue afectado y 100, que fue completamente destruído.

Antes de realizar los análisis estadísticos, para homogenizar las varianzas, los datos experimentales de control de malezas fueron transformados a su valor de arco seno $\%$, de acuerdo a lo que se recomienda en Gómez y Gómez (1984). Los análisis de varianza se efectuaron con los datos transformados y como prueba de comparación de medias se utilizó Tukey al 0,05. Sin embargo, por motivos de claridad, en los Cuadros del 5 al 10 se presentan los datos originales.

\section{RESULTADOS Y DISCUSIÓN}

Densidad de población de malezas. En 2001, en los lotes experimentales se presentaron cinco especies de malezas, pertenecientes a cuatro familias botánicas. La población de malezas al momento de la aplicación de los herbicidas era de 1.232.500 plantas/ha, siendo las especies dominantes la hierba ceniza (Lagascea mollis Cav.) y la flor amarilla (Baltimora recta L.), ambas de la familia Asteraceae, las cuales en conjunto ocuparon el 93,81\% de la población total de malezas (Cuadro 3). Por otro lado, en 2002, se tuvieron ocho especies de malezas, pertenecientes a ocho familias botánicas. Al

Cuadro 3. Especies de malezas presentes en los lotes experimentales y su densidad de población en 2001. Veracruz, México. 2001.

\begin{tabular}{lllr}
\hline Nombre común & \multicolumn{1}{c}{ Nombre científico } & \multicolumn{1}{c}{ Familia $^{1}$} & Población/ha $^{2}$ \\
\cline { 2 - 4 } Hierba ceniza & Lagascea mollis Cav. & Asteraceae & 755.000 \\
Flor amarilla & Baltimora recta L. & Asteraceae & 401.250 \\
Malva peluda & Malachra fasciata Jacq. & Malvaceae & 58.750 \\
Frijolillo & Senna obtusifolia (L.) Irwin \& Barneby & Caesalpiniaceae & 10.000 \\
Gusanillo & Acalypha alopecuroides Jacq. & Euphorbiaceae & 7.500 \\
Total & & & $\mathbf{1 . 2 3 2 . 5 0 0}$ \\
\hline
\end{tabular}

${ }^{1}$ Se utilizó el nombre de la familia botánica indicado por Villaseñor y Espinosa (1998).

2 Promedio de los experimentos de Crosser y Vaquero. 
momento de la aplicación de los herbicidas, la población de malezas era de 668.750 plantas/ha. La especie dominante fue el frijolillo [Senna obtusifolia (L.) Irwin \& Barneby] de la familia Caesalpiniaceae, que ocupó el $50,28 \%$ de la población total de malezas (Cuadro 4).

\section{Control de L. mollis}

Experimento con Crosser. A los 15 DDA, el control de L. mollis por el herbicida Crosser varió de 66,3 a $86,3 \%$, siendo esta respuesta directamente proporcional a las diferentes dosis evaluadas, aunque la dosis de 2 1/100 1 agua, solamente fue significativamente superior a la de 0,75 1/100 1 de agua. En esta época de evaluación, la dosis más alta de Full-Mina 4 proporcionó el mayor control de esta especie con cerca de $95 \%$.

En todos los tratamientos con herbicidas, a los 30 DDA se observaron incrementos en el control de L. mollis desde 3,7 hasta 15.3\%. Full-Mina 4, a 2 1/100 1 de agua, controló casi completamente a esta especie y también se tuvieron controles superiores al $95 \%$ con Esterón 47-M y la dosis más alta de Crosser, que tuvo controles significativamente mayores que los que se obtuvieron con las demás dosis de este herbicida. Finalmente, a los 60 DDA, los controles L. mollis, se mantuvieron o disminuyeron ligeramente, debido a que las plantas que no fueron eliminadas completamente desarrollaron nuevo follaje y/o hubo emergencia de algunas plantas después de los 30 DDA. A excepción de Crosser a 0,75 y 1 1/100 1 de agua, todos los tratamientos herbicidas tuvieron controles de L. mollis superiores al $80 \%$ (Cuadro 5).

Experimento con Vaquero. Este herbicida tuvo un control deficiente de L. mollis desde 0,75 hasta 1,5
1/100 1 de agua, con controles máximos de entre 40 y $62,5 \%$ a los 30 DDA. En esa época de evaluación, la dosis más alta de Vaquero, tuvo un control cercano al $80 \%$, pero éste se redujo a $71,3 \%$ a los 60 DDA. A su vez, los controles máximos proporcionados con las dosis alta y baja de Full-Mina 4 y con Esterón 47-M, fueron de 99, 88 y 95,3\%, respectivamente (Cuadro 6).

\section{Control de $B$. recta}

Experimento con Crosser. A los 15 DDA, se obtuvo un control total de $B$. recta con el herbicida Crosser a la dosis de 2 1/100 1 de agua, siendo estadísticamente semejante a los controles obtenidos con este mismo herbicida a 1; 1,25 y 1,5 1/100 l agua, así como con Full-Mina 4 a 1 y 2 1/100 1 de agua y Esterón 47-M a 1 1/100 1 agua. El herbicida Crosser a 0,75 1/100 1 agua fue el tratamiento que tuvo el control más bajo de esta especie, sin embargo, éste fue de 92\%. A los 30 y 60 DDA, todos los tratamientos herbicidas ofrecieron un control total de B. recta (Cuadro 7).

Experimento con Vaquero. A los 15 DDA, todos los tratamientos herbicidas mostraban un control de entre 90 y $100 \%$ de B. recta. Tanto Vaquero a 2 1/100 l de agua como Full-Mina 4 a la misma dosis, mostraban un control total de esta especie, mientras que los controles obtenidos con Full-Mina 4 a 1 1/100 1 de agua, Esterón 47-M a 1 1/100 1 de agua y Vaquero a 1.25 y 1,5 1/100 L de agua, variaron entre el 98,8 y 99,5\%, siendo todos los tratamientos antes mencionados estadísticamente semejantes entre sí. Vaquero a 0,75 y 1 1/100 1 de agua tuvieron los controles más bajos de $B$. recta, sin embargo, sus valores fueron de 90 y $95,5 \%$ respectivamente. A los 30 y 60 DDA, todos los tratamientos herbicidas ofrecieron un control total de B. recta (Cuadro 8).

Cuadro 4. Especies de malezas presentes en los lotes experimentales y su densidad de población en 2002. Veracruz, México. 2001.

\begin{tabular}{lllr}
\hline Nombre común & \multicolumn{1}{c}{ Nombre científico } & Familia & Población/ha \\
& & & \\
Frijolillo & Senna obtusifolia (L.) Irwin \& Barneby & Caesalpiniaceae & 336.250 \\
Vergonzosa & Mimosa pudica L. & Mimosaceae & 188.750 \\
Escobilla & Sida acuta Burm. f. & Malvaceae & 106.250 \\
Quelite & Amaranthus hybridus L. & Amaranthaceae & 12.500 \\
Cacahuatillo & Kallstroemia maxima (L.) Torr. \& A. Gray & Zygophyllaceae & 10.000 \\
Hierba mora & Solanum nigrum L. & Solanaceae & 7.500 \\
Golondrina & Chamaesyce hirta (L.) Millsp & Euphorbiaceae & 5.000 \\
Chilillo & Polanisia viscosa (L.) DC. & Capparaceae & 2.500 \\
Total & & & $\mathbf{6 6 8 . 7 5 0}$ \\
\hline
\end{tabular}

1 Se utilizó el nombre de la familia botánica indicado por Villaseñor y Espinosa (1998).

2 Promedio de los experimentos de Crosser y Vaquero. 
Cuadro 5. Control de Lagascea mollis (\%) con Crosser a los 15, 30 y 60 DDA. Veracruz, México. 2001.

\begin{tabular}{|c|c|c|c|c|}
\hline Tratamiento & $\begin{array}{c}\text { Dosis } \\
\text { (1/100 l agua) }\end{array}$ & 15 DDA & 30 DDA & 60 DDA \\
\hline 1. Crosser & 0,75 & $66,3 \mathrm{c}^{*}$ & $75,0 \mathrm{~d}$ & $70,0 \mathrm{f}$ \\
\hline 2. Crosser & 1 & $78,8 \mathrm{bc}$ & $83,8 \mathrm{~cd}$ & 78,8 ef \\
\hline 3. Crosser & 1,25 & $81,3 \mathrm{~b}$ & $85,0 \mathrm{~cd}$ & $83,8 \mathrm{def}$ \\
\hline 4. Crosser & 1,5 & $82,5 \mathrm{~b}$ & $86,3 \mathrm{~cd}$ & 86,3 cde \\
\hline 5. Crosser & 2 & $86,3 \mathrm{ab}$ & $95,5 \mathrm{ab}$ & 96,0 abc \\
\hline 6. Full-Mina 4 & 1 & $77,5 \mathrm{bc}$ & $92,8 \mathrm{bc}$ & $91,0 \mathrm{bcd}$ \\
\hline 7. Full-Mina 4 & 2 & $94,5 \mathrm{a}$ & 99,5 a & 99,5 a \\
\hline 8. Esterón 47-M & 1 & $86,3 \mathrm{ab}$ & $97,3 \mathrm{ab}$ & $96,3 \mathrm{ab}$ \\
\hline $\begin{array}{l}\text { 9. Testigo sin } \\
\text { aplicar }\end{array}$ & - & $0,0 \mathrm{~d}$ & $0,0 \mathrm{e}$ & $0,0 \mathrm{~g}$ \\
\hline
\end{tabular}

*Tukey $(0,05)$.

Cuadro 6. Control de Lagascea mollis (\%) con Vaquero a los 15, 30 y 60 DDA. Veracruz, México. 2001.

\begin{tabular}{|c|c|c|c|c|}
\hline Tratamiento & $\begin{array}{l}\text { Dosis } \\
00 \text { I agua) }\end{array}$ & 15 DDA & 30 DDA & 60 DDA \\
\hline 1. Vaquero & 0,75 & $30,0 \mathrm{~d}^{*}$ & $40,0 \mathrm{e}$ & $27,5 \mathrm{e}$ \\
\hline 2. Vaquero & 1 & $37,5 \mathrm{~d}$ & $50,0 \mathrm{de}$ & $37,5 \mathrm{de}$ \\
\hline 3. Vaquero & 1,25 & $40,0 \mathrm{~d}$ & $55,0 \mathrm{de}$ & 45,0 de \\
\hline 4. Vaquero & 1,5 & $42,5 \mathrm{~d}$ & $62,5 \mathrm{~d}$ & $47,5 \mathrm{~d}$ \\
\hline 5. Vaquero & 2 & $62,5 \mathrm{c}$ & $78,8 \mathrm{c}$ & $71,3 \mathrm{c}$ \\
\hline 6. Full-Mina 4 & 1 & $80,0 \mathrm{ab}$ & 88,0 bc & $85,0 \mathrm{bc}$ \\
\hline 7. Full-Mina 4 & 2 & 90,0 a & 99,0 a & 97,3 a \\
\hline 8. Esterón 47-M & 1 & $71,3 \mathrm{bc}$ & $95,3 \mathrm{ab}$ & $93,3 \mathrm{ab}$ \\
\hline 9. Testigo sin aplicar & - & $0,0 \mathrm{e}$ & $0,0 \mathrm{f}$ & $0,0 \mathrm{f}$ \\
\hline
\end{tabular}

*Tukey $(0,05)$.

\section{Control de S. obtusifolia}

Experimento con Crosser. A los 15 DDA, con la dosis mayor de Crosser, se obtuvo un control de $S$. $o b$ tusifolia significativamente más alto al proporcionado por el resto de los tratamientos herbicidas y solamente otros dos tratamientos: Esterón 47-M y Crosser a 1,5 1/100 1 de agua, tuvieron controles de esta especie superiores al 80\%. A los 30 DDA, el Crosser a partir de 1 1/100 1 de agua, así como la dosis alta de Full-Mina 4 eliminaron entre el 99 y $100 \%$ de las plantas de S. $o b$ tusifolia. El control más bajo fue proporcionado por Esterón 47-M, aunque éste fue superior a 90\%. A los 60 DDA, el Crosser prácticamente había eliminado las plantas de S. obtusifolia a partir de 1 1/100 l de agua; también se observó un 100\% de control de esta especie con Full-Mina 4, aplicada a la dosis alta (Cuadro 9).
Cuadro 7. Control de Baltimora recta (\%) con Crosser a los 15 , 30 y 60 DDA. Veracruz, México. 2001.

\begin{tabular}{llrlrr}
\hline Tratamiento & $\begin{array}{c}\text { Dosis } \\
\text { (l/100 l agua) }\end{array}$ & 15 DDA & 30 DDA & 60 DDA \\
\hline 1. Crosser & 0,75 & 92,0 & $\mathrm{~b}^{*}$ & 100,0 & 100,0 \\
2. Crosser & 1 & 97,3 & ab & 100,0 & 100,0 \\
3. Crosser & 1,25 & 98,8 ab & 100,0 & 100,0 \\
4. Crosser & 1,5 & 99,0 & a & 100,0 & 100,0 \\
5. Crosser & 2 & 100,0 a & 100,0 & 100,0 \\
6. Full-Mina 4 & 1 & 97,0 ab & 100,0 & 100,0 \\
7. Full-Mina 4 & 2 & 99,8 a & 100,0 & 100,0 \\
8. Esterón 47-M & 1 & 98,3 ab & 100,0 & 100,0 \\
9. Testigo sin aplicar & - & $0,0 \quad \mathrm{c}$ & 0,0 & 0,0 \\
\hline
\end{tabular}

*Tukey $(0,05)$.

Experimento con Vaquero. La acción de este herbicida sobre S. obtusifolia, fue más lenta que la que se observó con el herbicida Crosser a las mismas dosis de aplicación, ya que a los 15 DDA, el mayor control de esta especie, era de sólo 66,3\%. Sin embargo, entre los 15 y los 30 DDA, se tuvo un fuerte aumento en los controles, ya que en esta última época de evaluación, incluso con la dosis más baja de Vaquero, el control era de cerca de 95\%, el cual era estadísticamente semejante a los controles obtenidos con el resto de los tratamientos herbicidas. A los 60 DDA, el control más bajo obtenido con el Vaquero, fue de $97 \%$, similar al proporcionado por la dosis baja de Full-Mina 4. Con las otras dosis de Vaquero, S. obtusifolia fue completamente eliminada (Cuadro 10).

Para poder comparar la efectividad en el control de las malezas herbáceas de los herbicidas en desarrollo Crosser y Vaquero, con respecto a aquellos que se

Cuadro 8. Control de Baltimora recta (\%) con Vaquero a los 15, 30 y 60 DDA. Veracruz, México. 2001.

\begin{tabular}{lrrrrr}
\hline \multicolumn{1}{c}{ Tratamiento } & $\begin{array}{c}\text { Dosis } \\
(\mathbf{l} / \mathbf{1 0 0} \text { l agua })\end{array}$ & 15 DDA 30 DDA 60 DDA \\
\hline 1. Vaquero & 0,75 & 90,0 & $\mathrm{c}^{*}$ & 100,0 & 100,0 \\
2. Vaquero & 1 & 95,5 & $\mathrm{bc}$ & 100,0 & 100,0 \\
3. Vaquero & 1,25 & 99,0 & $\mathrm{ab}$ & 100,0 & 100,0 \\
4. Vaquero & 1,5 & 99,5 & $\mathrm{a}$ & 100,0 & 100,0 \\
5. Vaquero & 2 & 100,0 & $\mathrm{a}$ & 100,0 & 100,0 \\
6. Full-Mina 4 & 1 & 98,8 ab & 100,0 & 100,0 \\
7. Full-Mina 4 & 2 & 100,0 & $\mathrm{a}$ & 100,0 & 100,0 \\
8. Esterón 47-M & 1 & 99,3 a & 100,0 & 100,0 \\
9. Testigo sin aplicar & - & 0,0 & $\mathrm{~d}$ & 0,0 & 0,0 \\
\hline
\end{tabular}

*Tukey $(0,05)$. 
Cuadro 9. Control de Senna obtusifolia (\%) con Crosser a los 15, 30 y 60 DDA. Veracruz, México. 2001.

\begin{tabular}{lcccccccc}
\hline \multicolumn{1}{c}{ Tratamiento } & $\begin{array}{c}\text { Dosis } \\
\text { (I/100 l agua) }\end{array}$ & 15 DDA 30 DDA & 60 DDA \\
\hline 1. Crosser & 0,75 & 45,0 & $\mathrm{e}^{*}$ & 95,8 & $\mathrm{bc}$ & 97,0 & $\mathrm{bc}$ \\
2. Crosser & 1 & 62,5 & $\mathrm{~d}$ & 99,3 & $\mathrm{ab}$ & 99,5 & $\mathrm{ab}$ \\
3. Crosser & 1,25 & 66,3 & $\mathrm{~cd}$ & 99,5 & $\mathrm{ab}$ & 100,0 & $\mathrm{a}$ \\
4. Crosser & 1,5 & 83,8 & $\mathrm{~b}$ & 100,0 & $\mathrm{a}$ & 100,0 & $\mathrm{a}$ \\
5. Crosser & 2 & 97,5 & $\mathrm{a}$ & 100,0 & $\mathrm{a}$ & 100,0 & $\mathrm{a}$ \\
6. Full-Mina 4 & 1 & 47,5 & $\mathrm{e}$ & 97,5 & $\mathrm{abc}$ & 97,5 & $\mathrm{abc}$ \\
7. Full-Mina 4 & 2 & 76,3 & $\mathrm{bc}$ & 99,0 & $\mathrm{ab}$ & 100,0 & $\mathrm{a}$ \\
8. Esterón 47-M & 1 & 82,5 & $\mathrm{~b}$ & 93,3 & $\mathrm{c}$ & 95,8 & $\mathrm{c}$ \\
9. Testigo sin aplicar & - & 0,0 & $\mathrm{f}$ & 0,0 & $\mathrm{~d}$ & 0,0 & $\mathrm{~d}$ \\
\hline
\end{tabular}

*Tukey $(0,05)$.

utilizan en la actualidad, se debe tomar en cuenta la o las especies de maleza que se pretenden controlar; por ejemplo, si se tienen infestaciones de $B$. recta y $S$. $o b$ tusifolia, tanto el Crosser como el Vaquero, podrían competir favorablemente con Full-Mina 4 y Esterón 47-M, ya que incluso a 0,75 l/100 1 de agua, proporcionan un excelente control de las especies de maleza antes indicadas. Sin embargo, si el problema es con $L$. mollis, el Vaquero no podría recomendarse y se necesitarían de 1,5 a 21 de Crosser/100 1 de agua para controlarla, por lo que en este caso, pudiera ser más económico controlar esta maleza con Esterón 47-M o Full-Mina 4.

Cuadro 10. Control de Senna obtusifolia (\%) con Vaquero a los 15, 30 y 60 DDA. Veracruz, México. 2001.

\begin{tabular}{lccccccc}
\hline \multicolumn{1}{c}{ Tratamiento } & $\begin{array}{c}\text { Dosis } \\
(\mathbf{l} / \mathbf{1 0 0} \text { I agua) }\end{array}$ & 15 DDA & 30 DDA & $\mathbf{6 0}$ DDA \\
\hline 1. Vaquero & 0,75 & 32,5 & $\mathrm{e}^{*}$ & 94,5 & $\mathrm{ab}$ & 97,0 & $\mathrm{c}$ \\
2. Vaquero & 1 & 42,5 & $\mathrm{de}$ & 97,8 & $\mathrm{ab}$ & 99,5 & $\mathrm{ab}$ \\
3. Vaquero & 1,25 & 45,0 & $\mathrm{de}$ & 99,3 & $\mathrm{ab}$ & 100,0 & $\mathrm{a}$ \\
4. Vaquero & 1,5 & 58,8 & $\mathrm{bc}$ & 98,3 & $\mathrm{ab}$ & 100,0 & $\mathrm{a}$ \\
5. Vaquero & 2 & 66,3 & $\mathrm{~b}$ & 99,8 & $\mathrm{a}$ & 100,0 & $\mathrm{a}$ \\
6. Full-Mina 4 & 1 & 50,0 & $\mathrm{~cd}$ & 93,8 & $\mathrm{~b}$ & 97,3 & $\mathrm{bc}$ \\
7. Full-Mina 4 & 2 & 70,0 & $\mathrm{ab}$ & 97,5 & $\mathrm{ab}$ & 99,5 & $\mathrm{ab}$ \\
8. Esterón 47-M & 1 & 80,0 & $\mathrm{a}$ & 97,5 & $\mathrm{ab}$ & 100,0 & $\mathrm{a}$ \\
9. Testigo sin aplicar & - & 0,0 & $\mathrm{f}$ & 0,0 & $\mathrm{c}$ & 0,0 & $\mathrm{~d}$ \\
\hline
\end{tabular}

*Tukey $(0,05)$.

Toxicidad a los pastos. No se observaron daños tóxicos ni en el zacate Pangola ni en el Estrella de Africa con ninguno de los tratamientos evaluados, en ninguna de las fechas de evaluación.

\section{CONCLUSIONES}

Se obtuvo un buen control de Lagascea mollis con el herbicida Crosser aplicado a 1,5 y 2l/100 1 de agua, sin embargo el control de esta especie con el herbicida Vaquero no fue eficiente

Full-Mina 4 a 1 1/100 1 de agua tuvo un control de Lagascea mollis semejante al de Crosser a 2 1/ha.

Baltimora recta y Senna obtusifolia pueden ser controladas totalmente con los herbicidas Crosser y Vaquero, incluso con las dosis de 0,75 1/100 1 de agua.

A las dosis evaluadas, la aplicación de los herbicidas Crosser y Vaquero no ocasionaron toxicidad a los zacates Estrella de África y Pangola.

\section{LITERATURA CITADA}

AVILA C., J. M. 1988. Efecto de dos herbicidas y diesel sobre el control de mezquite (Prosopis juliflora) y huizache (Acacia farnesiana). In: I. Aguilar (ed.). Memorias IX Congreso Nacional de la Ciencia de la Maleza. Cd. Juárez, Chih. p. 231-236.

ENRÍQUEZ, J. F.; MELÉNDEZ, F.; BOLAÑOS, E. D. 1999. Tecnología para la producción y manejo de forrajes tropicales en México. INIFAP. CIRGOC. Campo Experimental Papaloapan. Libro Técnico Núm. 7. Veracruz, México. 262 p.

ESQUEDA E., V. A. 2000. Efecto del chapeo manual y dos herbicidas en el control de la maleza y la producción de forraje de zacate Pangola (Digitaria decumbens Stent.). In: F. Guevara (ed), Memorias XXI Congreso Nacional de la Ciencia de la Maleza. Morelia, Mich., México. p.112-117.

GÓMEZ, K. A.; GÓMEZ, A. A. 1984. Statistical procedures for agricultural research. 2nd. ed. New York, J. Wiley \& Sons. $680 \mathrm{p}$.

HERNÁNDEZ, J. O.; REICHERT, A. 1987. Evaluación de cinco herbicidas sobre el control de malezas en potreros de clima Af (c). In: I. Aguilar (comp.). Memorias VIII Congreso Nacional de la Ciencia de la Maleza. San Luis Potosí, S. L. P., México. p.123-127.

JUÁREZ, F. I.; CONTRERAS, J.; MONTERO, M. 2000. Determinación de la tasa de digestión de gramíneas tropicales en el estado de Veracruz. In: E. López, F. Alpírez, J.A. Cruz (comps.). Memorias XIII Reunión Científica Tecnológica Forestal y Agropecuaria Veracruz 2000. Veracruz, Ver., México. s.p.

KOPPEL, E. T.; ORTÍZ, G. A.; AVILA, A.; LAGUNES, J.; CASTAÑEDA, O. G.; LÓPEZ, I.; AGUILAR, U.; 
ROMÁN., H.; VILLAGÓMEZ, J. A.; AGUILERA, R.; QUIRÓZ, J.; CALDERÓN, R. C. 1999. Manejo de ganado bovino de doble propósito en el trópico. INIFAP. CIRGOC. Libro Técnico Núm. 5. Veracruz, México. 158 p.

MOTOOKA, P. S. 1986. Chemical weed control in tropical pastures. In: K. Moody (ed.). Weed control in tropical crops. Vol. II. Los Baños Laguna, Philippines: Weed Science Society of the Philippines. Southeast Asian Region Center for Graduate Study and Research in Agriculture. p. 9-54.

RADILLO, F.; NAVA, B. 2001. Evaluación de aplicación química y método de chapeo para control de Acacia farnesiana L. Willd en praderas. In: F. Guevara, F. Alpírez, J.A. Cruz (comps.). Memoria XXII Congreso Nacional de la Ciencia de la Maleza. Colima, Col., México. p. 56.
REICHERT P., A. 1986. El huisache de la parte central de Veracruz problemática y control. In: J.L. Gutiérrez (ed.). Resúmenes VII Congreso Nacional de la Ciencia de la Maleza. y VIII Congreso de la Asociación Latinoamericana de Malezas. Guadalajara, Jal., México. p. 194.

SILVA A., J. H.; BELTRÁN, R. G.; VALDÉZ, M. G. 1990. Estudio preliminar de plantas tóxicas presentes en los agostaderos de la zona norte del estado de Colima. In: SOMECIMA (ed.). Resúmenes XI Congreso Nacional de la Ciencia de la Maleza. Irapuato, Gto., México. p. 105.

VILLASEÑOR, J. L.; ESPINOSA, F. J. 1998. Catálogo de malezas de México. México. Universidad Nacional Autónoma de México. Consejo Nacional Consultivo Fitosanitario. Fondo de Cultura Económica. 449 p. 